\title{
A predictive study of multivariate factors affecting goal attainment of INR after anticoagulation in patients with nonvalvular atrial fibrillation
}

\author{
Cheng Chang ${ }^{1}$, Wenxu Pan ${ }^{2}$, Yuqing Wang ${ }^{2}$, Juying Li ${ }^{1}$, Lian Zhang ${ }^{1}$, Jun Jin ${ }^{2}$ \\ ${ }^{1}$ Department of Cardiovascular Medicine, Yibin First People's Hospital, Yibin, China; ${ }^{2}$ Department of Cardiovascular Medicine, Xinqiao Hospital \\ Affiliated to Army Military Medical University, Chonqing, China \\ Contributions: (I) Conception and design: C Chang, J Jin; (II) Administrative support: J Li, L Zhang; (III) Provision of study materials or patients: \\ C Chang, W Pan, Y Wang, J Li, J Jin; (IV) Collection and assembly of data: All authors; (V) Data analysis and interpretation: C Chang, Y Wang, L \\ Zhang, J Jin; (VI) Manuscript writing: All authors; (VII) Final approval of manuscript: All authors. \\ Correspondence to: Jun Jin. No. 83, Xinqiao Main Street, Shapingba District, Chongqing, China. Email: 2357576024@qq.com.
}

Background Non-valvular atrial fibrillation (NVAF) is a common type of AF, and patients with NVAF have a higher risk of ischemic stroke than non-AF patients. This study aims to investigate the goal attainment of international normalized ratio (INR) in patients with NVAF after anticoagulation therapy, and to analyze the risk factors that affect the goal attainment of INR.

Methods: NVAF patients who were admitted to our hospital from December 2019 to December 2020 and received anticoagulation therapy were selected as the research subjects. The INR goal attainment of patients was assessed, the risk factors affecting INR goal attainment were analyzed, and a ROC curve was drawn to evaluate the predictive value of risk factors for INR goal attainment in NVAF patients.

Results: After anticoagulation treatment, the INR of 42 cases reached the target (INR value $\geq 0.2$, the goal attainment group), and the INR of 74 cases did not reach the target (INR value $<2.0$, the non-goal attainment group). The age, mean platelet volume (MPV), platelet distribution width (PDW), and large platelet ratio (P-LCR) levels of patients in the goal attainment group were significantly lower than those in the non-goal attainment group, and the platelet count (PLT) level was higher than that of the non-goal attainment group $(\mathrm{P}<0.05)$. The results of multivariate logistic regression analysis showed that MPV, PDW, and P-LCR were independent risk factors that affected the failure in INR goal attainment in patients with NVAF after anticoagulation therapy. The ROC curve showed that the AUC values of MPV, PDW, and P-LCR were $0.711,0.748,0.867$, respectively, and the combined AUC was 0.876, which was higher than that of the single detection.

Conclusions: MPV, PDW, and P-LCR are important factors that affect the goal attainment of INR after anticoagulant therapy in NVAF patients. For patients with risk factors, clinicians can formulate a reasonable individualized anticoagulant drug regimen based on the above-mentioned index levels.

Keywords: Non-valvular atrial fibrillation (NVAF); international standardized ratio; anticoagulant therapy; influencing factors

Submitted Feb 05, 2021. Accepted for publication Apr 17, 2021.

doi: 10.21037/apm-21-549

View this article at: http://dx.doi.org/10.21037/apm-21-549 


\section{Introduction}

As a common type of arrhythmia, atrial fibrillation (AF) refers to the loss of regular and orderly atrial electrical activity, replaced by rapid and disordered waves of AF (1). Epidemiology shows that the global incidence of $\mathrm{AF}$ is approximately $1 \%$, while the prevalence of $\mathrm{AF}$ in China is approximately $0.77 \%$, and the incidence gradually increases with age. Non-valvular atrial fibrillation (NVAF) is a common type of AF, accounting for $65.2 \%$ of all AF patients (2), and patients with NVAF have a nearly 5 times higher risk of ischemic stroke than non-AF patients. The risk of stroke caused by AF can be reduced by effective anticoagulation therapy. At present, oral warfarin anticoagulation is the preferred treatment for $\mathrm{AF}$ stroke prevention, which can reduce the risk of $\mathrm{AF}$ thromboembolism by $62 \%$ without increasing bleeding complications (3). However, patients have poor treatment compliance, the treatment rate is not high, and the rate of reaching the standard of international normalized ratio (INR) compliance rate is also low (4). Herein, this study analyzed the goal attainment of INR in NVAF patients after anticoagulation treatment, and further explored the risk factors that affect INR goal attainment, so as to provide a reference for improving the goal attainment of INR in NVAF patients after anticoagulation treatment. We present the following article in accordance with the STARD reporting checklist (available at http://dx.doi.org/10.21037/ apm-21-549).

\section{Methods}

\section{General information}

NVAF patients admitted to our hospital from December 2019 to December 2020 who received warfarin anticoagulation therapy were selected as the study subjects. The inclusion criteria were as follows: (I) patients met the diagnostic criteria for NVAF (5); (II) patients with chronic persistent $\mathrm{AF}$ or $\mathrm{AF}$ duration $>3$ months; (III) patients with good compliance, who could actively cooperate with research and treatment; (IV) patients with complete clinical data without missing information. The exclusion criteria were as follows: (I) patients with severe heart, liver, and kidney dysfunction; (II) patients with connective tissue disease; (III) patients with a history of blood transfusion in the past 6 months and a history of surgery within 3 months; (IV) patients with various acute and chronic infections and trauma.
Finally, a total of 116 patients (64 males and 52 females) with NVAF were included, aged 58-83 years, with an average age of $72.26 \pm 10.54$ years. The course of disease was $1-9$ years, with an average of $3.47 \pm 1.03$ years. All procedures performed in this study involving human participants were in accordance with the Declaration of Helsinki (as revised in 2013). The informed consent was taken from all the patients. This study was approved by the Ethics Committee of the First Hospital of Yibin (2021-015).

\section{Research content}

\section{General data}

General information of patients including gender, age, smoking history, course of disease, and comorbid diseases (cardiovascular disease, diabetes, cerebrovascular disease) were recorded.

\section{Laboratory indexes}

For every patient, $5 \mathrm{~mL}$ of venous blood was drawn on an empty stomach the next morning after the patient was admitted to the hospital, and the blood cell indicators and blood biochemical indicators were detected with an automatic blood cell analyzer and biochemical analyzer. Blood cell indicators included white blood cells (WBC), red blood cells (RBC), platelet count (PLT), platelet specific product (PCT), average platelet volume (MPV), platelet distribution width (PDW), and large platelet ratio (P-LCR). Blood biochemical indicators included aspartate aminotransferase (AST), alanine aminotransferase (ALT), serum creatinine (SCr), total cholesterol (TC), triacylglycerol (TG), high-density lipoprotein cholesterol (HDL-C), low-density lipoprotein cholesterol (LDL-C), and coagulation function indicators, such as INR, D-dimer (D-Dimer), and fibrinogen (FIB).

\section{Calculation of the CHADS2 score and the CHA2DS2- VASC score of AF patients}

The total score for CHADS2 was 6 points, and the total score for CHA2DS2-VASC was 9 points (6). Anticoagulant therapy was considered if the total score was $\geq 2$ points.

\section{Observation indicators}

Firstly, INR goal attainment was analyzed. Secondly, patients were grouped according to INR goal attainment, and the risk factors that affect the goal attainment of INR were analyzed. Evaluation criteria for the goal attainment 
Table 1 Comparison of the general information between the two groups

\begin{tabular}{|c|c|c|c|c|c|}
\hline Index & & Goal attainment group $(n=42)$ & Non-goal attainment group $(n=74)$ & $t / \chi^{2}$ & $\mathrm{P}$ \\
\hline Gender & Female & $27(64.29 \%)$ & $35(47.30 \%)$ & & \\
\hline Age (year) & & $69.58 \pm 10.05$ & $75.19 \pm 10.14$ & 2.873 & 0.005 \\
\hline Hypertension & Yes & $23(54.76 \%)$ & $45(60.81 \%)$ & 0.404 & 0.525 \\
\hline \multirow[t]{2}{*}{ Diabetes } & Yes & $6(14.29 \%)$ & $9(12.16 \%)$ & 0.107 & 0.743 \\
\hline & No & $36(85.71 \%)$ & $65(87.84 \%)$ & & \\
\hline \multirow[t]{2}{*}{ Smoking history } & Yes & $10(23.81 \%)$ & $30(40.54 \%)$ & 3.320 & 0.068 \\
\hline & No & $32(76.19 \%)$ & $44(59.46 \%)$ & & \\
\hline \multirow[t]{2}{*}{ Coronary heart disease } & Yes & $15(35.71 \%)$ & $31(41.89 \%)$ & 0.427 & 0.513 \\
\hline & No & 27 (64.29\%) & $43(58.11 \%)$ & & \\
\hline
\end{tabular}

of INR (7) were as follows: according to the NVAF anticoagulation treatment guidelines of the American College of Cardiology/American Heart Association/ European Society of Cardiology ACC/AHA/ESC, the INR goal attainment value was 1.6-2.5 for patients aged $>75$ years old and with an increased risk of bleeding complications; for high-risk patients aged $<75$ years old, primary prevention and secondary prevention were conducted for patients who have had stroke, and the INR goal attainment value was 2.0 to 3.0; the value of related independent risk factors for predicting whether the INR of NVAF patients met the standard was analyzed.

\section{Statistical analysis}

The data in this study were statistically analyzed by SPSS 22.0 software (IBM, New York, USA). The measurement data were described by the mean \pm standard deviation $\left(\bar{x}_{ \pm s}\right)$, and comparisons between groups were performed using the $t$ test. The count data was expressed as $\mathrm{n}(\%)$ and analyzed by the chi-squared $\left(\chi^{2}\right)$ test. Logistic regression was used to analyze the risk factors affecting the INR goal attainment of NVAF patients after anticoagulation treatment. The ROC curve was used to analyze the predictive value of the relevant independent risk factors for the INR goal attainment of NVAF patients. Results with $\mathrm{P}<0.05$ were considered to be statistically significant.

\section{Results}

\section{INR compliance}

After anticoagulation therapy, the INR values of the 116 patients with NVAF ranged from 0.79 to 3.34 , with an average of $1.07 \pm 0.52$. There were 42 cases with INR reaching the standard (INR value $\geq 0.2$, the goal attainment group), and 74 patients with INR values lower than the standard (INR value $<2.0$, the non-goal attainment group).

\section{Comparison of general information between the two groups}

The mean age of the patients in the goal attainment group was significantly lower than that in the non-goal attainment group $(\mathrm{P}<0.05)$, and there were no significant differences in other general indexes between the two groups $(\mathrm{P}>0.05$, Table 1).

\section{Comparison of laboratory indicators, total CHADS2 scores, and CHA2DS2-VASC scores between the two groups}

The PLT level of the goal attainment group was significantly higher than that of the non-goal attainment group, and the levels of MPV, PDW, P-LCR, and LDL-C were significantly lower than those of the non-goal attainment group. The differences between the groups were statistically significant $(\mathrm{P}<0.05)$, while the differences in other indicators between the two groups were not 
Table 2 Comparison of laboratory indicators, total CHADS2 scores, and CHA2DS2-VASC scores between the two groups

\begin{tabular}{|c|c|c|c|c|}
\hline Indicators & Goal attainment group $(n=42)$ & Non-goal attainment group $(n=74)$ & $\mathrm{t}$ & $P$ \\
\hline $\operatorname{RBC}\left(\times 10^{12} / \mathrm{L}\right)$ & $4.15 \pm 0.58$ & $4.37 \pm 0.88$ & 1.450 & 0.150 \\
\hline $\operatorname{PLT}\left(\times 10^{9} / \mathrm{L}\right)$ & $198.01 \pm 58.07$ & $172.24 \pm 64.75$ & 2.137 & 0.035 \\
\hline MPV (fL) & $9.78 \pm 1.65$ & $11.87 \pm 1.74$ & 6.333 & 0.001 \\
\hline P-LCR (\%) & $26.97 \pm 6.17$ & $35.17 \pm 6.28$ & 6.801 & 0.001 \\
\hline $\operatorname{ALT}(U / L)$ & $27.47 \pm 10.05$ & $26.57 \pm 9.33$ & 0.486 & 0.628 \\
\hline $\mathrm{SCr}(\mu \mathrm{mol} / \mathrm{L})$ & $90.47 \pm 33.65$ & $89.66 \pm 42.47$ & 0.106 & 0.916 \\
\hline D-Dimer (mg/L) & $1.47 \pm 0.56$ & $1.49 \pm 0.48$ & 0.203 & 0.840 \\
\hline $\mathrm{TG}(\mathrm{mmol} / \mathrm{L})$ & $1.16 \pm 0.62$ & $1.21 \pm 0.57$ & 0.440 & 0.661 \\
\hline HDL-C (mmol/L) & $1.05 \pm 0.33$ & $1.07 \pm 0.36$ & 0.296 & 0.768 \\
\hline LDL-C (mmol/L) & $2.04 \pm 0.69$ & $3.11 \pm 0.76$ & 7.748 & 0.001 \\
\hline CHADS2 (point) & $2.25 \pm 1.41$ & $2.47 \pm 1.11$ & 1.016 & 0.312 \\
\hline CHA2DS2-VASC (point) & $3.58 \pm 1.72$ & $3.69 \pm 1.66$ & 0.339 & 0.736 \\
\hline
\end{tabular}

WBC, white blood cells; RBC, red blood cells; PLT, platelet count; MPV, mean platelet volume; PDW, platelet distribution width; P-LCR, platelet-large cell rate; ALT, alanine aminotransferase; SCr, serum creatinine; FIB, fibrinogen; TC, total cholesterol; TG, triacylglycerol; HDL-C, high-density lipoprotein cholesterol; LDL-C, low-density lipoprotein cholesterol; CHADS2: C (congestive) H (hypertension), A (age), D (diabetes mellitus), S2 (prior stroke or TIA); CHA2DS2-VASC referred to congestive heart failure, hypertension, age $\geq 75 \mathrm{y}$ (doubled), diabetes mellitus, stroke (doubled)-vascular disease, age 65-74 and sex category (female).

significant $(\mathrm{P}>0.05$, Table 2).

\section{Multi-factor analysis of non-goal attainment of INR in patients with NVAF after anticoagulation therapy}

Multivariate logistic regression analysis was performed by taking whether the INR value reached the goal as the dependent variable, and taking indicators of age, PLT, MPV, PDW, P-LCR, and LDL-C as independent variables. Results demonstrated that indicators such as MPV, PDW, and P-LCR were independent risk factors of non-goal attainment of INR in patients with NVAF after anticoagulation therapy $(\mathrm{P}<0.01$, Table 3).

\section{The predictive value of MPV, PDW, and P-LCR for non- goal attainment of INR in patients with NVAF}

ROC results showed that the areas under the curve (AUC) of MPV, PDW, and P-LCR were 0.711, 0.748, and 0.867, respectively, and the AUC of combined detection was 0.876 , which was significantly higher than that of the single detection (Table 4 and Figure 1).

\section{Discussion}

In recent years, stroke has become the main cause of death from cerebrovascular diseases in China, and the incidence of ischemic stroke in AF patients is gradually increasing. In addition, $\mathrm{AF}$ patients are often accompanied by enhanced coagulation activity, pre-thrombosis, and thromboembolism. Oral anticoagulant therapy with rivaroxaban, dabigatran, warfarin and other drugs and ablation of NVAF are common methods for clinical treatment of AF. Among them, anticoagulant therapy is more common, which can effectively reduce the risk of stroke in $\mathrm{AF}$ patients. However, due to their own factors, the anticoagulant effect is not significant in a small number of patients after oral anticoagulants $(8,9)$. Studies have shown that warfarin treatment shows a definite 
Table 3 Multivariate analysis of the risk factors of non-goal attainment of INR in patients with NVAF after anticoagulation therapy

\begin{tabular}{lccccc}
\hline Factor & Regression coefficient & Standard error & Wald value & $\mathrm{P}$ value & OR (95\% Cl) \\
\hline Age (old vs. young) & 0.154 & 0.785 & 1.595 & 0.147 & $1.166(0.250-5.434)$ \\
PLT (high vs. low) & 0.189 & 0.266 & 0.456 & 0.125 & $1.208(0.717-2.035)$ \\
MPV (high vs. low) & 0.546 & 0.216 & 7.509 & $<0.01$ & $1.726(1.130-2.636)$ \\
PDW (high vs. low) & 0.685 & 0.274 & 10.035 & $<0.01$ & $1.984(1.159-3.394)$ \\
P-LCR (high vs. low) & 1.264 & 0.344 & 10.233 & $<0.01$ & $3.540(1.804-6.947)$ \\
LDL-C (high vs. low) & 0.198 & 0.154 & 1.084 & 0.078 & $1.219(0.901-1.648)$ \\
\hline
\end{tabular}

INR, international normalized ratio; NVAF, non-valvular atrial fibrillation; PLT, platelet count; MPV, mean platelet volume; PDW, platelet distribution width; P-LCR, platelet-large cell rate; LDL-C, low-density lipoprotein cholesterol.

Table 4 Predictive value of MPV, PDW, and P-LCR for non-goal attainment of INR in patients with NVAF

\begin{tabular}{lcccc}
\hline Predictive factor & Sensitivity & Specificity & AUC & $95 \% \mathrm{Cl}$ \\
\hline MPV & 0.741 & 0.712 & 0.711 & $0.571-0.851$ \\
PDW & 0.847 & 0.731 & 0.748 & $0.623-0.873$ \\
P-LCR & 0.867 & 0.805 & 0.867 & $0.775-0.958$ \\
Combination & 0.954 & 0.851 & 0.876 & $0.787-0.964$ \\
\hline
\end{tabular}

MPV, mean platelet volume; PDW, platelet distribution width; P-LCR, platelet-large cell rate; INR, international normalized ratio; NVAF, nonvalvular atrial fibrillation; AUC, area under the curve; $\mathrm{Cl}$, confidence interval.

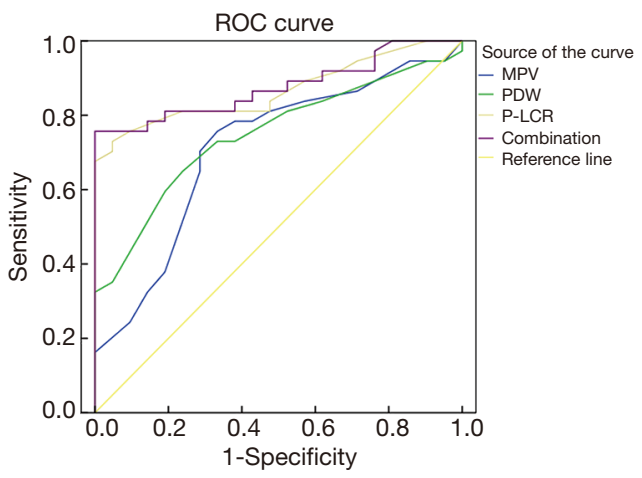

Figure 1 ROC curve of MPV, PDW, and P-LCR for nongoal attainment of INR in patients with NVAF. ROC, receiver operating characteristic curve; MPV, mean platelet volume; PDW, platelet distribution width; P-LCR, platelet-large cell rate; INR, international normalized ratio; NVAF, non-valvular atrial fibrillation.

effect on $\mathrm{AF}$ patients, and different doses and frequencies of warfarin treatment have a certain relationship with the occurrence of ischemic stroke. Warfarin has a definite effect in the treatment of patients with $\mathrm{AF}$, and can reduce the incidence of stroke, mortality, and the incidence of composite endpoint events (10). Oral warfarin is of great significance in preventing cerebral infarction in $\mathrm{AF}$ patients (11). INR is a commonly used indicator to monitor the dose and safety of warfarin. Barrios et al. (12) pointed out that although warfarin shows a good therapeutic effect, it takes a long time to reach the ideal INR, and the maintenance time of the target range of INR is short. Ali et al.'s study on the recommended anti-thrombotic strategies after valve replacement suggested that patients with mechanical or biological valve replacement should use warfarin for anticoagulation after surgery, and the INR value should be maintained at 2.0-3.0 to reach anticoagulation standards (13). Therefore, in order to ensure the INR compliance rate of anticoagulation therapy, it is of great significance to analyze the factors that affect INR goal attainment.

This study analyzed the relationships between various clinical factors and INR goal attainment, and found that MPV, PDW, and P-LCR were independent risk factors for INR non-goal attainment after anticoagulation in AF patients. We found that patients with high MPV, PDW, and P-LCR were prone to having low rates of INR goal attainment, suggesting that in addition to strengthening anticoagulation therapy for $\mathrm{AF}$ patients in the future, 
it is necessary to focus on people with abnormalities in the above indicators, which is consistent with the report by Elvira Ruiz (14). However, a foreign study by Gue et al. (15) has shown that the age and smoking history of AF patients are risk factors that affect the non-goal attainment of INR after anticoagulation therapy. There are discrepancies with the results reported in this study, which may be related to factors such as sample size and regional population differences. MPV, PDW, and P-LCR are common platelet indexes, which can effectively reflect the function of platelets. Routine detection of platelet indexes is of great significance for the risk assessment of embolism in $\mathrm{AF}$ patients (16). Because large platelets are more easily activated than normal-sized platelets, MPV can reflect platelet function indirectly, as MPV is related to platelet size. Research from Seiffge et al. (17) shows that the MPV level of AF patients is negatively correlated with coronary blood flow velocity. The higher the MPV level, the higher the risk of acute coronary events. Studies abroad have also shown that elevated MPV level is associated with blood stasis in the left atrium of AF patients. Furthermore, MPV, and especially PDW, are important markers of platelet activation, and the combination of the 2 indexes is of great value for judging whether the blood is hypercoagulable (18). Platelet indexes such as MPV, PDW, and PCT reflect the functional status of the body's coagulation system, and is closely related to thrombosis.

In order to further analyze the value of MPV, PDW, and P-LCR for predicting whether the INR meets the standard, an ROC curve was used in this study. ROC curves are widely used in the performance evaluation of medical diagnostic tests, which can obtain multiple pairs of true positive rates (TPR) and false positive rates (FPR) by changing the diagnostic boundary. The curve is drawn with FPR as the abscissa, and with TPR as the ordinate $(19,20)$. The diagnostic value of the diagnostic test is reflected by calculating and comparing the AUC. The results of this study showed that the sensitivity and specificity of MPV, PDW, and P-LCR for predicting whether INR meets the standard were high, and the combined detection of the three indicators showed even higher sensitivity and specificity, with a greater value of AUC. These results indicated that monitoring the platelet function of AF patients is of great significance for assessing whether anticoagulation meets the goal and adjusting the dose of anticoagulant drugs. However, since this study is a single-center study and the sample size of included cases is limited, the conclusion of this study needs to be confirmed by further studies with a large sample size across multiple centers. In addition, due to the fact that this study was a single-center study with a limited number of enrolled patients, subsequent studies with a large sample size and multiple centers are still needed to confirm the conclusion of this work

In summary, MPV, PDW, and P-LCR are important factors that affect whether the INR reaches the standard after anticoagulation treatment in NVAF patients. For patients with risk factors, a reasonable and individualized anticoagulant drug regimen can be made clinically based on the detection of the above index levels.

\section{Acknowledgments}

Funding: None.

\section{Footnote}

Reporting Checklist: The authors have completed the STARD reporting checklist. Available at http://dx.doi.org/10.21037/ apm-21-549

Data Sharing Statement: Available at http://dx.doi. org/10.21037/apm-21-549

Conflicts of Interest: All authors have completed the ICMJE uniform disclosure form (available at http://dx.doi. org/10.21037/apm-21-549). The authors have no conflicts of interest to declare.

Ethical Statement: The authors are accountable for all aspects of the work in ensuring that questions related to the accuracy or integrity of any part of the work are appropriately investigated and resolved. All procedures performed in this study involving human participants were in accordance with the Declaration of Helsinki (as revised in 2013). The informed consent was taken from all the patients. This study was approved by the Ethics Committee of the First Hospital of Yibin (2021-015).

Open Access Statement: This is an Open Access article distributed in accordance with the Creative Commons Attribution-NonCommercial-NoDerivs 4.0 International License (CC BY-NC-ND 4.0), which permits the noncommercial replication and distribution of the article with the strict proviso that no changes or edits are made and the original work is properly cited (including links to both the formal publication through the relevant DOI and the license). 
See: https://creativecommons.org/licenses/by-nc-nd/4.0/.

\section{References}

1. Pappas MA, Barnes GD, Vijan S. Personalizing Bridging Anticoagulation in Patients with Nonvalvular Atrial Fibrillation-a Microsimulation Analysis. J Gen Intern Med 2017;32:464-70.

2. Lamberts M, Staerk L, Olesen JB, et al. Major Bleeding Complications and Persistence With Oral Anticoagulation in Non-Valvular Atrial Fibrillation: Contemporary Findings in Real-Life Danish Patients. J Am Heart Assoc 2017;6:e04517.

3. Kawabata M, Goya M, Sasaki T, et al. Left Atrial Appendage Thrombi Formation in Japanese Non-Valvular Atrial Fibrillation Patients During Anticoagulation Therapy - Warfarin vs. Direct Oral Anticoagulants. Circ J 2017;81:645-51.

4. Caro J, Navada S. Safety of anticoagulation in patients with atrial fibrillation and MDS/AML complicated by thrombocytopenia: An unresolved challenge: Can they be managed? A report of three cases and literature review. Am J Hematol 2018;93:E112-E114.

5. Gibson CM, Smith CB, Davis S, et al. Assessment of Apixaban Prescribing Patterns for Nonvalvular Atrial Fibrillation in Hospitalized Patients. Ann Pharmacother 2018;52:54-9.

6. McAlister FA, Wiebe N, Hemmelgarn BR. Time in therapeutic range and stability over time for warfarin users in clinical practice: a retrospective cohort study using linked routinely collected health data in Alberta, Canada. BMJ Open 2018;8:e016980.

7. Camm AJ, Turpie AGG, Hess S, et al. Outcomes after catheter ablation and cardioversion in patients with nonvalvular atrial fibrillation: results from the prospective, observational XANTUS study. Europace 2018;20:e87-e95.

8. Pappas MA, Vijan S, Rothberg MB, et al. Reducing age bias in decision analyses of anticoagulation for patients with nonvalvular atrial fibrillation - A microsimulation study. PLoS One 2018;13:e0199593.

9. Laube ES, Yu A, Gupta D, et al. Rivaroxaban for Stroke Prevention in Patients With Nonvalvular Atrial Fibrillation and Active Cancer. Am J Cardiol 2017;120:213-7.

10. Wald R, Dorian P, Harel Z. Benefits and Risks of Anticoagulation in Dialysis Patients With Nonvalvular Atrial Fibrillation: Navigating Through Darkness. J Am Coll Cardiol 2020;75:286-8.

11. Escobar C, Borrás X, Bover Freire R, et al. A Delphi consensus on the management of oral anticoagulation in patients with non-valvular atrial fibrillation in Spain: ACOPREFERENCE study. PLoS One 2020;15:e0231565.

12. Barrios V, Cinza-Sanjurjo S, Gavín O, et al. Cost and burden of poor anticoagulation control with vitamin $\mathrm{K}$ antagonists in patients with nonvalvular atrial fibrillation in Spain. Rev Esp Cardiol (Engl Ed) 2020. [Epub ahead of print]. doi: 10.1016/j.rec.2020.06.033.

13. Ali M, Rigopoulos AG, Mammadov M, et al. Systematic review on left atrial appendage closure with the LAmbre device in patients with non-valvular atrial fibrillation. BMC Cardiovasc Disord 2020;20:78.

14. Elvira Ruiz G, Caro Martínez C, Flores Blanco PJ, et al. Effect of concomitant antiplatelet therapy in patients with nonvalvular atrial fibrillation initiating non-vitamin $\mathrm{K}$ antagonists. Eur J Clin Invest 2019;49:e13161.

15. Gue YX, Inoue N, Spinthakis N, et al. Thrombotic Profile and Oral Anticoagulation in Asian and Non-Asian Patients With Nonvalvular Atrial Fibrillation. J Am Coll Cardiol 2019;74:2822-4.

16. Lee SR, Choi EK, Kwon S, et al. Oral Anticoagulation in Asian Patients With Atrial Fibrillation and a History of Intracranial Hemorrhage. Stroke 2020;51:416-23.

17. Seiffge DJ, Werring DJ, Paciaroni M, et al. Timing of anticoagulation after recent ischaemic stroke in patients with atrial fibrillation. Lancet Neurol 2019;18:117-26.

18. Fawzy AM, Lip GYH. Anticoagulation in elderly patients with nonvalvular atrial fibrillation: A balancing act. Heart Rhythm 2019;16:38-40.

19. Weernink MGM, Vaanholt MCW, Groothuis-Oudshoorn CGM, et al. Patients' Priorities for Oral Anticoagulation Therapy in Non-valvular Atrial Fibrillation: a Multicriteria Decision Analysis. Am J Cardiovasc Drugs 2018;18:493-502.

20. Lobos-Bejarano JM, Castellanos Rodríguez A, Barrios $\mathrm{V}$, et al. Influence of renal function on anticoagulation control in patients with non-valvular atrial fibrillation taking vitamin $\mathrm{K}$ antagonists. Int J Clin Pract 2017. doi: 10.1111/ijcp.12974.

(English Language Editor: C. Betlazar-Maseh)

Cite this article as: Chang C, Pan W, Wang Y, Li J, Zhang L, Jin J. A predictive study of multivariate factors affecting goal attainment of INR after anticoagulation in patients with nonvalvular atrial fibrillation. Ann Palliat Med 2021;10(4):45324538. doi: 10.21037/apm-21-549 